\title{
Non-inertial effects in reactions of astrophysical interest
}

\author{
C. A. Bertulani, J.T. Huang, and P. G. Krastev* \\ Department of Physics, Texas A\&M University, Commerce, TX 75429, USA
}

(Dated: January 14, 2019)

\begin{abstract}
We discuss the effects of non-inertial motion in reactions occurring in laboratory, stars, and elsewhere. It is demonstrated that non-inertial effects due to large accelerations during nuclear collisions might have appreciable effects nuclear and atomic transitions. We also explore the magnitude of the corrections induced by strong gravitational fields on nuclear reactions in massive, compact stars, and the neighborhood of black holes.
\end{abstract}

PACS numbers: 26.20.+f, 26.50.+x, 24.10.-i

\section{INTRODUCTION}

Extremely large accelerations occur when atomic nuclei collide. For instance, two lead nuclei in a head on collision with a center of mass kinetic energy of $500 \mathrm{MeV}$, reach a closest distance of $19.4 \mathrm{fm}$ before they bounce back and move outward. At this distance each nucleus accelerates with an intriguing $\sim 10^{27} \mathrm{~m} / \mathrm{s}^{2}$. Very few other physical situations in the Universe involve nuclei undergoing such large accelerations, usually related to astrophysical objects, as in the vicinity of neutron stars and black holes, where huge gravitational fields exist. In this article we explore the effects of large accelerations and large gravitational fields, and their possible influence on nuclear reactions in the laboratory and in astrophysical environments. Nuclear reactions are crucial for the formation of stellar structures and their rates could be affected by various factors. To our knowledge, the effect of large gravitational fields on nuclear reaction rates in stars has not been considered so far.

As mentioned in the previous paragraph, atomic and nuclear systems undergo large accelerations during reactions. The effect of acceleration is observed in terms of excitations followed by decay of these systems. If we consider two-body reactions, there are two systems of reference which are often used to describe the effects of the collision: (a) the center-of-mass $(\mathrm{cm})$ system of the two nuclei and (b) the system of reference of the excited nucleus. System (b) is appropriate to use when the intrinsic properties of the excited nucleus is described in some nuclear model. A typical example is the case of Coulomb excitation. One assumes that the nuclei scatter and their $\mathrm{cm}$ wave functions are described by Coulomb waves due to the Coulomb repulsion between the nuclei. Then one considers the residual effect of the Coulomb potential on the motion of the nucleons inside the nuclei. This is done by expanding the Coulomb potential in multipoles and using the high order terms (higher than first order) as a source of the excitation process. In this approach one illustrates the privileged role of the $\mathrm{cm}$ of the nuclear system: the net effect of the external forces is to

*Current address: San Diego State University, Department of Physics, 5500 Campanile Dr., San Diego, CA 92182-1233

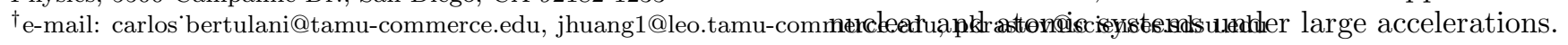

(i) accelerate all the particles together, along with the $\mathrm{cm}$ of the system, and (ii) to change the intrinsic quantum state of the system through the spatial variation of the interaction within the system. Thus the theoretical treatment of accelerated many-body systems is well under control in non-relativistic dynamics.

In the non-relativistic case, the separation of variables into intrinsic motion and relative motion between the $\mathrm{cm}$ of each nucleus is a simple algebraic procedure. A problem arises when one wants to extend the method to describe intrinsic excitations of relativistic many-body systems. Very few works exist in the literature addressing this problem. The reason is that for nuclear reactions in the laboratory, the effect is expected to be very small, a common belief which must be tested. Another other reason is that in stellar environments where the gravitational fields are large, huge pressures develop, "crushing" atoms, stripping them from their electrons, and ultimately making nuclei dissolve into their constituents. Effects of nuclear excitation are not relevant in the process. But, on the other hand, nuclear reactions are crucial for the formation of stellar structures and their rates could be affected by minor effects such as those explored in this article.

Nuclei participating in nuclear reactions in a gaseous phase of a star follow inertial trajectories between collisions with other nuclei. Such trajectories are "free fall" trajectories in which all particles within the nucleus have the same acceleration. That is surely true in the nonrelativistic case, but not in the relativistic one because retardation effects lead to corrections due to the nuclear sizes. The central problem here is the question regarding the definition of the center of mass of a relativistic many body system. We have explored the literature of this subject and found few cases in which this problem is discussed. Based on their analysis we show that relativistic effects introduce small corrections in the Lagrangian of a many-body system involving the magnitude of their acceleration. We follow Refs. [1, 2, 3], with few modifications, to show that a correction term proportional to the square of the acceleration appears in the frame of reference of the accelerated system. To test the relevance of these corrections, we make a series of applications to 


\section{HAMILTONIAN OF AN ACCELERATED MANY-BODY SYSTEM}

Starting with a Lagrangian of a free particle in an inertial frame and introducing a coordinate transformation into an accelerated frame with acceleration $\mathcal{A}$, a "fictitious force" term appears in the Lagrangian when written in coordinates fixed to the accelerated frame. Thus, in an accelerated system the Lagrangian $L$ for a free particle can be augmented by a (non-relativistic) interaction term of the form $-m \mathcal{A} z$, that is

$$
L=-m c^{2}+\frac{1}{2} m v^{2}-m \mathcal{A} z,
$$

where $z$ is the particle's coordinate along the direction of acceleration of the reference frame [1].

In the relativistic case, the first step to obtain the Lagrangian of a many body system in an accelerated frame is to setup an appropriate measure of space-time in the accelerated frame, i.e. one needs to find out the proper space-time metric. The free-particle action $S=-m c \int d s$ requires that $d s=\left(c-v^{2} / 2 c+\mathcal{A} z\right) d t$, which can be used to obtain $d s^{2}$. To lowest order in $1 / c^{2}$ one gets

$d s^{2}=c^{2}\left(1+\frac{\mathcal{A} z}{c^{2}}\right)^{2} d t^{2}-d x^{2}-d y^{2}-d z^{2}=g_{\mu \nu} d \xi^{\nu} d \xi^{\mu}$,

where $\mathbf{v} d t=d \mathbf{r}$ was used, with $d \xi^{\mu}=(c d t, d x, d y, d z)$ and $g_{\mu \nu}=\left(g_{00},-1,-1,-1\right), g_{00}=\left(1+\mathcal{A} z / c^{2}\right)^{2}$. The indices $\mu$ run from 0 to 3. Eq. (2) gives a general form for the metric in an accelerated system. This approach can be found in standard textbooks (see, e.g. ref. [1], $\S$ 87).

From the definition for the Hamiltonian, $H=\mathbf{p} \cdot \mathbf{v}-L$, with $\mathbf{p}=\partial L / \partial \mathbf{v}=m \mathbf{v} / \sqrt{g_{00}-v^{2} / c^{2}}$, and using the action with the metric of Eq. (2), after a straightforward algebra one finds

$$
H=\frac{g_{00} m c^{2}}{\sqrt{g_{00}-\frac{v^{2}}{c^{2}}}}=c \sqrt{g_{00}\left(p^{2}+m^{2} c^{2}\right)} .
$$

Expanding $H$ in powers of $1 / c^{2}$, one obtains

$H=\frac{p^{2}}{2 m}\left(1-\frac{p^{2}}{4 m^{2} c^{2}}\right)+m \mathcal{A} z\left(1+\frac{p^{2}}{2 m^{2} c^{2}}\right)+\mathcal{O}\left(\frac{1}{c^{4}}\right)$.

This Hamiltonian can be applied to describe a system of particles with respect to a system of reference moving with acceleration $\mathcal{A}$, up to order $1 / c^{2}$. For an accelerated nucleus the obvious choice is the $\mathrm{cm}$ system of the nucleus. But then the term carrying the acceleration correction averages out to zero in the center of mass, as one has $\left(\sum_{i} m_{i} \mathcal{A} z_{i}=0\right)$. There is an additional small contribution of the acceleration due to the term proportional to $p^{2}$. Instead of exploring the physics of this term, one has to account for one more correction as explained below.

The above derivation of the Hamiltonian for particles in accelerated frames does not take into account that the definition of the $\mathrm{cm}$ of a collection of particles is also modified by relativity. This is not a simple task as might seem at first look. There is no consensus in the literature about the definition of the $\mathrm{cm}$ of a system of relativistic particles. The obvious reason is the role of simultaneity and retardation. Ref. [2] examines several possibilities. For a system of particles it is found convenient to define the coordinates $q^{\mu}$ of the center of mass as the mean of coordinates of all particles weighted with their dynamical masses (energies). The relativistic (covariant) generalization of center of mass is such that the coordinates $q^{\mu}$ must satisfy the relation [2]

$$
P^{0} q^{\mu}=\sum_{i} p_{i}^{0} z_{i}^{\mu}
$$

where the coordinates of the $i$ th particle with respect to the center of mass are denoted by $z_{i}^{\mu}$ and the total momentum vector by $P^{\mu}=\sum_{i} p_{i}^{\mu}$. Ref. [2] chooses eq. (5) as the one that is most qualified to represent the definition of $\mathrm{cm}$ of a relativistic system, which also reduces to the non-relativistic definition of the center of mass. We did not find a better discussion of this in the literature and we could also not find a better way to improve on this definition.

The above definition, Eq. (5), leads to the compact form, to order $1 / c^{2}$,

$$
\begin{aligned}
\sum_{i} \frac{m_{i} \mathbf{r}_{i}}{\sqrt{g_{00}-\frac{v_{i}^{2}}{c^{2}}}} & =\sum_{i} m_{i} \mathbf{r}_{i}\left(1+\frac{v_{i}^{2}}{2 c^{2}}-\frac{z_{i} \mathcal{A}}{c^{2}}+\mathcal{O}\left(\frac{1}{c^{4}}\right)\right) \\
& =0
\end{aligned}
$$

where $\mathbf{r}_{i}=\left(x_{i}, y_{i}, z_{i}\right)$ is the coordinate and $v_{i}$ is the velocity of the ith particle with respect to the $\mathrm{cm}$.

For a system of non-interacting particles the condition in Eq. (6) implies that, along the direction of motion,

$$
\sum_{i} \mathcal{A} m_{i} z_{i}=-\sum_{i} \mathcal{A} m_{i} z_{i}\left(\frac{v_{i}^{2}}{2 c^{2}}-\frac{z_{i} \mathcal{A}}{c^{2}}\right) .
$$

Hence, the Hamiltonian of Eq. (4) for a collection of particles becomes

$H=\sum_{i} \frac{p_{i}^{2}}{2 m_{i}}\left(1-\frac{p_{i}^{2}}{4 m_{i}^{2} c^{2}}\right)+\frac{\mathcal{A}^{2}}{c^{2}} \sum_{i} m_{i} z_{i}^{2}+U\left(r_{i}\right)+\mathcal{O}\left(\frac{1}{c^{4}}\right)$,

where we have added a scalar potential $U\left(r_{i}\right)$, which would represent a (central) potential within an atom, a nucleus, or any other many-body system.

Notice that the term proportional to $-m \mathcal{A} z$ completely disappears from the Hamiltonian after the relativistic treatment of the $\mathrm{cm}$. This was also shown in Ref. [3]. It is important to realize that non-inertial effects will also carry modifications on the interaction between the particles. For example, if the particles are charged, there will be relativistic corrections (magnetic interactions) which need to be added to the scalar potential $U\left(r_{i}\right)=\sum_{j \neq i} Q_{i} Q_{j} /\left|\mathbf{r}_{i}-\mathbf{r}_{j}\right|$. As shown in Ref. [3] , the full treatment of non-inertial effects together with relativistic corrections will introduce additional terms proportional to $\mathcal{A}$ and $\mathcal{A}^{2}$ in the Hamiltonian of Eq. (8), to 
order $1 / c^{2}$. Thus, a more detailed account of non-inertial corrections of a many-body system requires the inclusion of $\mathcal{A}$-corrections in the interaction terms, too. We refer the reader to Ref. [3] where this is discussed in more details. Here we will only consider the consequences of the acceleration correction term in Eq. (8),

$$
H_{n i n}=\frac{\mathcal{A}^{2}}{c^{2}} \sum_{i} m_{i} z_{i}^{2}
$$

\section{REACTIONS IN STARS}

Nuclei interacting in a plasma or undergoing pycnonuclear reactions in a lattice can experience different accelerations, allowing for an immediate application of Eq. (9). But in order to use this equation to measure changes induced by the gravitational fields in stars, we assume that one can replace $\mathcal{A}$ by a local gravitational field, $g$. This assumption requires a few comments at this point. If we consider two nuclei participating in a nuclear reaction in a star, they are, most likely, in a gaseous phase following inertial trajectories in between collisions. The effect of gravity is to modify slightly the inertial trajectories of the two nuclei due to the difference in the gravitational field strength in their initial and final positions. Thus the best way to study the reaction problem is to calculate reaction rates in terms of a local metric at a point within the star. This metric can be deduced from General Relativity at the reaction observation point. To first-order one can also use Eq. (2), which is shown in Ref. [1] to describe particles in a gravitational field. Here instead, we will adopt the Hamiltonian of Eq. (8) as representative of the same problem. Here we will not attempt to prove the equality between the two procedures, and several other issues (e.g., time-dependence of accelerations, modification of interactions in presence of a gravitational field, etc.), leaving this for future studies. Our goal here is to estimate the magnitude of the gravitational field which could produce sizable "non-inertial corrections" and study physical cases where such corrections might be important and could change appreciably the reaction rates and/or the internal structure of many body systems.

\section{A. Nuclear fusion reactions}

Nuclear fusion reactions in stars proceed at low energies, e.g., of the order of $10 \mathrm{KeV}$ in our Sun [4, 5]. Due to the Coulomb barrier, it is extremely difficult to measure the cross sections for charged-particle-induced fusion reactions at laboratory conditions. The importance of small effects such as the correction of Eq. (9) in treating fusion reactions is thus clear because the Coulomb barrier penetrability depends exponentially on any correction. To calculate the effect of the term given by Eq. (9) we use, for simplicity, the WKB penetrability factor

$$
P(E)=\exp \left[-\frac{2}{\hbar} \int_{R_{N}}^{R_{C}} d r|p(r)|\right]
$$

where $p(r)$ is the (imaginary) particle momentum inside the repulsive barrier. The corrected fusion reaction is given by

$$
\sigma=\sigma_{C} \cdot \mathcal{R}
$$

where $\sigma_{C}$ is the Coulomb repulsion cross section and $\mathcal{R}=P_{\text {corr }}(E) / P(E)$ is the correction due to Eq. (9). The non-inertial effect is calculated using $|p(r)|=$ $\sqrt{2 m\left[V_{C}(r)-E\right]}$ and

$$
\left|p_{\text {corr }}(r)\right|=\sqrt{2 m\left[V_{C}(r)+\frac{\mathcal{A}^{2} m r^{2}\left\langle\cos ^{2} \theta\right\rangle}{c^{2}}-E\right]}
$$

where $\left\langle\cos ^{2} \theta\right\rangle=1 / 2$ averages over orientation and the Coulomb potential is given by $V_{C}=Z_{1} Z_{2} e^{2} / r$. In order to assess the magnitude of the acceleration $\mathcal{A}$ for which its effect is noticeable, we consider a proton fusion reaction with a $Z=17$ nucleus (chlorine) at $E=0.1 \mathrm{MeV}$. This is a typical fusion reaction in stellar sites of interest. For this energy, we get $R_{C}=Z_{1} Z_{2} e^{2} / E=245 \mathrm{fm}$ and take $R_{N}=3.2 \mathrm{fm}$.

As we see in Fig. 11 the effect of acceleration becomes visible for accelerations of the order $g=\mathcal{A}=$ $10^{-7} c^{2} / R_{C} \approx 4 \times 10^{27} \mathrm{~m} / \mathrm{s}^{2}$, which is about 26 orders of magnitude larger than the acceleration due to gravity on Earth's surface and 15 orders of magnitude larger than the one at the surface of a neutron star (assuming $M_{n s}=M_{\odot}$ and $R_{n s}=10 \mathrm{~km}$ ). It appears that the effect is extremely small in stellar environments of astrophysical interest where nuclear fusion reactions play a role. Such large gravitational fields would only be present in the neighborhood of a black-hole. Under such extreme conditions nuclei are likely to disassemble, as any other structure will.

\section{B. Atomic transitions}

As an example in atomic physics, we consider the energy of the $2 \mathrm{p}_{1 / 2}$ level in hydrogen which plays an important role in the Lamb shift and probes the depths of our understanding of electromagnetic theory. We calculate the energy shift of the $2 \mathrm{p}_{1 / 2}$ level within the first-order perturbation theory and we get

$$
\Delta E_{n i n}^{2 p_{1 / 2}}=\left\langle 2 p_{1 / 2}\left|H_{n i n}\right| 2 p_{1 / 2}\right\rangle=\frac{24 a_{H}^{2} \mathcal{A}^{2} m_{e}}{c^{2}},
$$

where $a_{H}=\hbar^{2} / m_{e} e^{2}=0.529 \AA$. One should compare this value with the Lamb splitting which makes the $2 \mathrm{p}_{1 / 2}$ state slightly lower than the $2 \mathrm{~s}_{1 / 2}$ state by $\Delta E_{L a m b}=$ $4.372 \times 10^{-6} \mathrm{eV}$. One gets $\Delta E_{n i n}^{2 p_{1 / 2}} \simeq \Delta E_{\text {Lamb }}$ for $\mathcal{A} \simeq$ $10^{21} \mathrm{~m} / \mathrm{s}^{2}$, which is 9 orders of magnitude larger than gravity at the surface of a neutron star. Thus, even for tiny effects in atomic systems, the effect would only be noticeable for situations in which electrons are bound in atoms. 


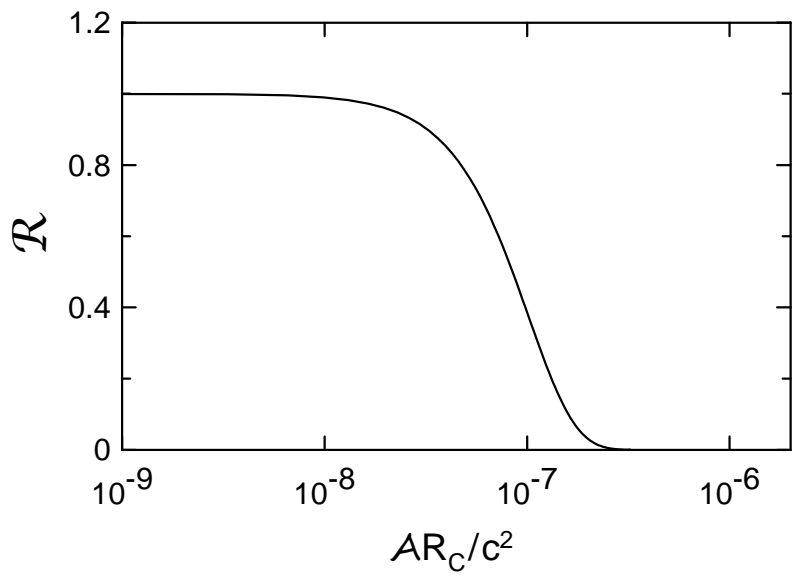

FIG. 1: Suppression factor due to the non-inertial effects, $\mathcal{R}$, for fusion reactions of protons on chlorine at $E=0.1 \mathrm{MeV}$, and as a function of the gravitational field (in dimensionless units).

\section{REACTIONS IN THE LABORATORY}

The logical conclusion from the last section is that it is very unlikely that non-inertial effects due to gravitational fields are of relevance in stars. Nowhere, except in the vicinity of a black-hole, accelerations are of order of $\sim 10^{20} \mathrm{~m} / \mathrm{s}^{2}$, which would make the effect noticeable. However, there is another way to achieve such large accelerations and that is nothing else but the huge accelerations which occur during nuclear reactions. For example, for a nuclear fusion reaction, at the Coulomb radius (distance of closest approach, $R_{C}$ ) the acceleration is given by

$$
\mathcal{A}_{C}=\frac{Z_{1} Z_{2} e^{2}}{R_{C}^{2} m_{0}}
$$

where $m_{0}=m_{N} A_{1} A_{2} /\left(A_{1}+A_{2}\right)$ is the reduced mass of the system and $m_{N}$ is the nucleon mass. For typical values, $E=1 \mathrm{MeV}, Z_{1}=Z_{2}=10$, and $A_{1}=A_{2}=$ 20, one obtains $R_{C}=Z_{1} Z_{2} e^{2} / E=144 \mathrm{fm}$ and $\mathcal{A}_{C}=$ $6.2 \times 10^{25} \mathrm{~m} / \mathrm{s}^{2}$. This is the acceleration that the $\mathrm{cm}$ of each nucleus would have with respect to the laboratory system.

As we discussed in the introduction, the $\mathrm{cm}$ of the excited nucleus is the natural choice for the reference frame. This is because it is easier to adopt a description of atomic and nuclear properties in the $\mathrm{cm}$ frame of reference. Instead, one could also chose the $\mathrm{cm}$ of the colliding particles. This later (inertial) system makes it harder to access the acceleration effects, as one would have to boost the wave functions to an accelerated system, after calculating it in the inertial frame. This is a more difficult task. Therefore we adopt the $\mathrm{cm}$ reference frame of the excited nucleus, using the Hamiltonian of section II. This Hamiltonian was deduced for a constant acceleration. If the acceleration is time-dependent, the metric of Eq. (2) also changes. Thus, in the best case scenario, the Hamiltonian of Eq. (8) can be justified in an adiabatic situation in which the relative velocity between the many-body systems is much smaller than the velocity of their constituent particles with respect to their individual center of masses. If we accept this procedure, we can study the effects of accelerated frames on the energy shift of states close to threshold, as well as on the energy location of low-lying resonances.

\section{A. Reactions involving halo nuclei}

The nuclear wave-function of a (s-wave) loosely-bound, or "halo", state can be conveniently parameterized by

$$
\Psi \simeq \sqrt{\frac{\alpha}{2 \pi}} \frac{\exp (-\alpha r)}{r},
$$

where the variable $\alpha$ is related to the nucleon separation energy through $S=\hbar^{2} \alpha^{2} / 2 m_{N}$. In first order perturbation theory the energy shift of a halo state will be given by

$$
\Delta E_{n i n}^{N}=\left\langle\Psi\left|H_{n i n}\right| \Psi\right\rangle=\frac{1}{8 S}\left(\frac{Z_{1} Z_{2} e^{2} \hbar}{R_{C}^{2} m_{0} c}\right)^{2}
$$

Assuming a small separation energy $S=100 \mathrm{keV}$, and using the same numbers in the paragraph after Eq. (14), we get $\Delta E_{n i n}^{N}=0.024 \mathrm{eV}$, which is very small, except for states very close to the nuclear threshold, i.e. for $S \rightarrow 0$. But the effect increases with $Z^{2}$ for symmetric systems (i.e. $Z_{1}=Z_{2}=A_{1} / 2$ ). It is thus of the order of $\Delta E_{n i n}^{N}=1-10 \mathrm{eV}$ for larger nuclear systems.

There might exist situations where this effect could be present. For instance, the triple-alpha reaction which bridges the mass $=8$ gap and forms carbon nuclei in stars relies on the lifetime of only $10^{-17} \mathrm{~s}$ of ${ }^{8} \mathrm{Be}$ nuclei. It is during this time that another alpha-particle meets ${ }^{8}$ Be nuclei in stars leading to the formation of carbon nuclei. This lifetime corresponds to an energy width of only $5.57 \pm 0.25 \mathrm{eV}$ [6]. As the third alpha particle approaches ${ }^{8} \mathrm{Be}$, the effects of linear acceleration will be felt in the reference frame of ${ }^{8} \mathrm{Be}$. This will likely broaden the width of the ${ }^{8}$ Be resonance (which peaks at $E_{R}=91.84 \pm 0.04$ $\mathrm{KeV}$ ) and consequently its lifetime. However, this line of thought could be wrong if one assumes that the third alpha particle interacts individually with each of the two alpha particles inside ${ }^{8} \mathrm{Be}$, and that the effects of acceleration internal to the ${ }^{8} \mathrm{Be}$ nucleus arise from the different distances (and thus accelerations) between the third alpha and each of the first two. To our knowledge, this effect has not been discussed elsewhere and perhaps deserves further investigation, if not for this particular reaction maybe for other reactions of astrophysical interest involving very shallow nuclear states.

\section{B. Nuclear transitions}

Many reactions of astrophysical interest are deduced from experimental data on nucleus-nucleus scattering. 
Important information on the position and widths of resonances, spectroscopic factors, and numerous other quantities needed as an input for reaction network calculations in stellar modeling are obtained by the means of nuclear spectroscopy using nuclear collisions in the laboratory. During the collision the nuclei undergo huge acceleration, of the order of $\mathcal{A} \simeq 10^{28} \mathrm{~m} / \mathrm{s}^{2}$. Hence, non-inertial effects will be definitely important.

A simple proof of the statements above can be obtained by studying the Coulomb excitation. The simplest treatment that one can use in the problem is a semi-classical calculation. The probability of exciting the nucleus to a state $f$ from an initial state $i$ is given by

$$
a_{i f}=-\frac{i}{\hbar} \int V_{i f} e^{i \omega t} d t
$$

where $\omega=\left(E_{f}-E_{i}\right) / \hbar$, is the probability amplitude that there will be a transition $\mathrm{i} \rightarrow \mathrm{f}$. The matrix element $V_{i f}=\int \Psi_{f}^{*} V \Psi_{i} d \tau$ contains a potential $V$ of interaction between the nuclei. The square of $a_{i f}$ measures the transition probability from $i$ to $f$ and this probability should be integrated along the trajectory.

A simple estimate could be obtained in the case of the excitation of a initial, $J=0$, state of a deformed nucleus to an excited state with $J=2$ as a result of a head on collision with scattering angle of $\theta=180^{\circ}$. The perturbation $V$ is due to the interaction of the charge $Z_{1} e$ of the projectile (one of the two nuclei) with the quadrupole moment of the target (of the other) nucleus. This quadrupole moment should work as an operator that acts between the initial and final states. One finds that $V=Z_{1} e^{2} Q_{i f} / 2 r^{3}$, with

$$
Q_{i f}=e_{i}^{2}\left\langle\Psi_{f}^{*}\left|3 z^{2}-r^{2}\right| \Psi_{i}\right\rangle \simeq e_{i}^{2}\left\langle\Psi_{f}^{*}\left|z^{2}\right| \Psi_{i}\right\rangle,
$$

where $e_{i}$ is the effective charge of the transition.

The amplitude is then written as

$$
a_{i f}=\frac{Z_{1} e^{2} Q_{i f}}{2 i \hbar} \int \frac{e^{i \omega t}}{r^{3}} d t
$$

At $\theta=180^{\circ}$ the separation $r$, the velocity $v$, the initial velocity $v_{0}$ and the distance of closest approach $s$, are related by $v=d r / d t= \pm_{0} v_{0}(1-s / r)$, which is obtained from energy conservation. Furthermore, if the excitation energy is small, we can assume that the factor $e^{i \omega t}$ in Eq. (19) does not vary much during the time that the projectile is close to the nucleus. Then the remaining integral is easily solved by substitution and one gets

$$
a_{i f}=\frac{4 Z_{1} e^{2} Q_{i f}}{3 i \hbar v_{0} s^{2}} .
$$

Following the same procedure as above, we can calculate the contribution of the Hamiltonian of Eq. (9). In this case, $\mathcal{A}=Z_{1} Z_{2} e^{2} / m_{0} r^{2}$ and the equivalent potential $V$ is given by

$$
V_{\text {nin }}=\left(\frac{Z_{1} Z_{2} e^{2}}{m_{0}}\right)^{2} \frac{X m_{N}}{c^{2} r^{4}}
$$

where we assume that $X$ nucleons participates in the transition. One then finds

$$
a_{i f}^{n i n}=\left(\frac{Z_{1} Z_{2} e^{2}}{m_{0}}\right)^{2} \frac{32 X m_{N} Q_{i f}}{15 i s^{3} \hbar v_{0} c^{2}} .
$$

The ratio between the two transition probabilities is

$$
\left|\frac{a_{i f}^{n i n}}{a_{i f}}\right|^{2}=\left(\frac{8 X m_{N} Z_{1} Z_{2}^{2} e^{2}}{5 s m_{0}^{2} c^{2}}\right)^{2} .
$$

Applying eq. 23 to the lead-lead collision at $500 \mathrm{MeV}$, as mentioned in the introduction, we find $\left|a_{i f}^{n i n} / a_{i f}\right|^{2}=$ $(0.0093 X)^{2}$. This yields very small results for the relative importance of non-inertial effects in single particle transitions $(X \simeq 1)$, but can become appreciable for the excitation of collective states such as the giant resonances, for which $X \gg 1$. This result is intriguing to say the least. We think that it deserves more studies, assuming that the physics of non-inertial effects described in section II is right. We have made a preliminary study of theses effects in the excitation of giant resonances in relativistic heavy ion collisions using Eq. (9) which seem to confirm this statement.

\section{Electron screening of fusion reactions}

In laboratory measurements of nuclear fusion reactions one has found enhancements of the cross sections due to the presence of atomic electrons. This screening effect leads to an enhancement in the astrophysical S-factor, or cross section:

$$
S_{l a b}(E)=f(E) S(E)=\exp \left[\frac{\pi \eta \Delta E}{E}\right] S(E),
$$

where $\eta(E)=Z_{1} Z_{2} e^{2} / \hbar v$, and $v$ is the relative velocity between the nuclei. The energy $\Delta E$ is equal to the difference between the electron binding energies in the $\left(Z_{1}+Z_{2}\right)$-system and in the target atom $\left(Z_{2}\right)$. For light nuclei it is of the order of $100 \mathrm{eV}$, enhancing fusion cross sections even for fusion energies of the order of $100 \mathrm{KeV}$. For more details we refer the interested reader to Ref. [7].

An intriguing fact is that this simple estimate, which is an upper value for $\Delta E$, fails to reproduce the experimental data for a series of cases. In Ref. [8] several small effects, ranging from vacuum polarization to the emission of radiation, have been considered but they cannot explain the experimental data puzzle. Besides vacuum polarization, atomic polarization is one of the largest effects to be considered (among all other small effects [8]).

Non-inertial corrections contribute to polarization potential

$$
V_{p o l}=-\sum_{n \neq 0} \frac{\left|\left\langle 0\left|H_{n i n}\right| n\right\rangle\right|^{2}}{E_{n}-E_{0}} .
$$

An estimate based on hydrogenic wave functions for the atom yields

$$
V_{p o l}(r) \simeq-\frac{1}{E_{n 0}}\left(\frac{Z_{1} Z_{2} e \hbar}{m_{0} c}\right)^{4} \frac{\exp (-2 \alpha r)}{r^{4}}
$$


Assuming $\alpha \cong 1 / a_{H}, E_{n 0}=E_{n}-E_{0} \cong 10 \mathrm{eV}$ and using Eqs. (10) and (11) to calculate the modification of the fusion cross sections due to this effect, we find the cross section for $\mathrm{D}(\mathrm{d}, \mathrm{p}) \mathrm{T}$ and ${ }^{6} \mathrm{Li}(d, \alpha){ }^{4} \mathrm{He}$ can increase by up to $10 \%$. This is surprising compared with the smaller values reported on Table 1 of Ref. [8]. It is not a very accurate calculation as it relies on many approximations. But it hints for a possible explanation of the difference between the experimental and theoretical values of $\Delta E$, as discussed in Ref. [7].

In stars, reactions occur within a medium rich in free electrons. The influence of dynamic effects of these electrons was first mentioned in Ref. [9] and studied in Ref. [10]. The underlying assumption is that the DebyeHueckel approximation, based on a static charged cloud, does not apply for fast moving nuclei. In fact, most of the nuclear fusion reactions occur in the tail of the Maxwell-Boltzmann distribution. For these nuclear velocities Ref. [10] finds that an appreciable modification of the Debye-Hueckel theory is necessary. One has to add to this finding the fact that the nuclei get very strongly accelerated as they approach each other, and this increases further the deformation of the Debye-Hueckel cloud.

\section{CONCLUSIONS}

In summary, assuming that the Hamiltonian for a system of particles moving in an accelerated frame contains a correction term of the form given by Eq. (9), we have explored the non-inertial effects for a limited set of nuclear reactions in stars and in the laboratory. These results are somewhat surprising and present a challenge to our understanding of accelerated many-body systems.
In the case of stellar environments, we have shown that only in the neighborhood of black-holes would noninertial effects become relevant. But then the whole method adopted here is probably not rigorous enough, as one may have to use the full machinery of general relativity. Nonetheless, it is very unlikely (and perhaps unimportant, except maybe for science-fiction-like timetraveling) that internal structures of any object is of any relevance when it is extremely close to a black-hole.

The apparent reason for the appearance of non-inertial effects in many-body systems is that the non-inertial term of Eq. (9) only appears when relativistic corrections are included, what has precluded its consideration in previous studies, especially for reactions that are thought to be fully non-relativistic such as fusion reactions in stars. The main question is whether the relativistic definition of the center of mass, through Eq. (5) as proposed by Pryce in Ref. 2] contains the right virtue of describing correctly the center of mass frame of relativistic manybody systems.

Even in the case of high energies nuclear collisions the intrinsic structure of the nuclei are sometimes an important part of the process under study. Fictitious forces will appear in this system which might not average out and appreciably influence the structure or transition under consideration. It is surprising that, for a reason not quite understood, this effect has been overseen in the literature so far.

\section{Acnowledgments}

This work was partially supported by the U.S. DOE grants DE-FG02-08ER41533 and DE-FC02-07ER41457 (UNEDF, SciDAC-2), the NSF grant PHY0652548, and the Research Corporation under Award No. 7123.
[1] L.D. Landau and E.M. Lifshitz, Classical theory of fields (Second Edition), Pergamon Press, p.286

[2] M. H. L. Pryce, Proc. Roy. Soc. A195, 62 (1948).

[3] M.P. Fewell, Nucl. Phys. A425 (1984) 373.

[4] D.D. Clayton, Principles of Stellar Evolution and Nucleosynthesis, McGraw-Hill, New York, 1968.

[5] C. Rolfs, W.S. Rodney, Cauldrons in the Cosmos, University of Chicago Press, Chicago, IL, 1988.
[6] D. R. Tilley et al., Nucl. Phys. A745, 155 (2004).

[7] C. Rolfs, Prog. Part. Nucl. Phys. 46, 23 (2001).

[8] A.B. Balantekin, C.A. Bertulani and M.S. Hussein, Nucl. Phys. A 627, 324 (1997).

[9] H.E. Mitler, Ap. J. 212, 513 (1977).

[10] C. Carraro, A. Schäffer and S.E. Koonin, Ap. J. 331, 565 (1988). 